Juliet Lodge, Daniel Nagel:

\title{
Introductory Remarks to the Ethical Issues of Networked Toys
}

Sunni the plush gummy bear is silently sitting on the bookshelf in the dorm room of three-year-old Hannah. Sunni has a green dress, a fluffy body, big eyes with beautiful eyelashes and a built-in camera with Wi-Fi connection. Hannah is sound asleep while the cameras humming motor keeps adjusting to monitor Hannah's breathing rhythm and to count phases of potential apnea. At the same time, a high-resolution picture of Hannah is transmitted to both her parent's screen in the living room and a remotely accessible server in case that Hannah's parents go out for dinner. The latter is only accessible through a secured password, or so the parents hope.

A settlement between the FTC and VTECH, a manufacturer of connected toys, earlier this year showed that any connection is only as good as its weakest link, which in this case was the poor safeguarding of the collected sensitive data by VTECH. ${ }^{1}$ VTECH operates Kid Connect, a messaging tool for minors, where parents can control the settings and thus the contacts their children have. In order to provide this service VTECH collects a surprisingly conclusive amount of data from both parents and their children. VTECH claimed to only store the respective data encrypted. They, however, failed to prevent hackers from gaining access to the data including pictures of children, data about their age and their home addresses. VTECH and FTC settled on a fine of $650,000.00 \$$ as well as on yearly audits of the realization of a comprehensive data protection program. Other reports unfortunately do not sound more reassuring and include inter alia a loss of voice recordings taken by toys, ${ }^{2}$ Bluetooth back-doors for interactive dolls, ${ }^{3}$ as well as snooping via smartwatches. ${ }^{4}$

While some states and toymakers have reacted, ${ }^{5}$ the best first step to approach this is to take a step back and have a closer look at the underlying issues.

\section{Networked toys - Artificial guardians for little princesses or demonic plastic princes?}

Networked toys dominate the shelves in toy stores at a time when neither their real benefits nor their potentially latent dangers have been fully explored. Do hyper-connected toys transform the relationship between adults, the child and its environment? Do they shape their minds and predispose them to seek convenience and speedy responses rather than rely on their own autonomous capacities for critical thought?

Questions such as who really is in control arise, both of the toys - parents, third parties or even the toddlers themselves - and of data (including biometrics) that might be collected for unclear purposes and opaque

\footnotetext{
${ }^{1}$ https://www.ftc.gov/news-events/press-releases/2018/01/electronic-toy-maker-vtech-settles-ftc-allegations-it-violated

${ }^{2}$ Lorenzo Franceschi-Bicchierai, Internet of Things Teddy Bear Leaked 2 Million Parent and Kids Message Recordings, https://motherboard.vice.com/en_us/article/pgwean/internet-of-things-teddy-bear-leaked-2-million-parent-and-kids-message-recordings

${ }^{3}$ Bundesnetzagentur zieht Kinderpuppe "Cayla" aus dem Verkehr, Press Release of 17 February 2017 https://www.bundesnetzagentur.de/SharedDocs/Downloads/DE/Allgemeines/Presse/Pressemitteilungen/2017/27012017_cayla.pdf?_blo $\mathrm{b}=$ publicationFile\&v $=2$

${ }^{4}$ Bundesnetzagentur geht gegen Kinderuhren mit Abhörfunktion vor, Press Release of 17 November 2017, https://www.bundesnetzagentur.de/SharedDocs/Downloads/DE/Allgemeines/Presse/Pressemitteilungen/2017/17112017_Verbrauchersch utz.pdf?_blob=publicationFile\&v=2

${ }^{5}$ Cf. E.g. Germany banning smartwatches for children (see Fn 4) or Mattel stopping the production of a toy AI device, Haley Tsukayama, Mattel Has Canceled Plans for a Kid-Focused AI Device That Drew Privacy Concerns, Wash. Post (Oct. 4, 2017),

https://www.washingtonpost.com/news/the-switch/wp/2017/10/04/mattel-has-an-ai-device-to-soothe-babies-experts-are-begging-themnot-to-sell-it.
} 
destinations. For what specific or linkable purpose and above all where and to whom is data transmitted? What ethical considerations should be addressed?

Is there an actual benefit for the children themselves? Do hyper connected devices and robo-toys teach them how to handle technology or does it erode their capacity for autonomous reflection as speed and convenience are prioritised in their on-line and off-line worlds? Do such toys presage fundamental transformation of childhood and the imagined and physical worlds?

Ulrich Gasper takes the lead in adressing these important questions in this issue by shedding light on the impact of networked toys in the playground, while Rocco Panetta and Federico Satore depict the legal backdrop which serves as a basis for any evaluation of such toys. Finally, Isabelle Moeller answers critical questions on the qualification and use of biometrics and artificial intelligence in respect to children. 